\title{
Arteriovenous fistula imitating myocardial ischaemia on electrocardiogram
}

\author{
Paweł Moskal ${ }^{1}$, Łukasz Klima ${ }^{2}$, Marek Jastrzębski ${ }^{1}$, Danuta Czarnecka ${ }^{1}$ \\ ${ }^{1} 1^{\text {st }}$ Department of Cardiology, Interventional Electrocardiology, and Hypertension, Jagiellonian University Medical College, Krakow, Poland \\ ${ }^{2} 1^{\text {st }}$ Department of Cardiology, Interventional Electrocardiology, and Hypertension, University Hospital, Krakow, Poland
}

A 32-year-old man with end-stage renal disease was admitted to a neurological department due to peripheral one-sided facial paralysis. At night, he developed slight dyspnoea but denied any chest pain. To rule out acute coronary syndrome (ACS), electrocardiography (ECG) was performed and serum troponin I was measured. ECG (Fig. 1A) showed prominent ST-segment elevation in leads I and $\mathrm{aVL}$ and ST-segment depressions in leads V1-V6. The level of high sensitive troponin I was elevated on first measurement $(47 \mathrm{ng} / \mathrm{L}$; norm $<19 \mathrm{ng} / \mathrm{L})$ and the patient was transferred to the cardiac intensive care unit with a preliminary diagnosis of ACS. Follow-up ECG revealed no ST-segment changes (Fig. 1B) and troponin I levels did not show significant dynamics (46.8 ng/L and $39.3 \mathrm{ng} / \mathrm{L}$ after $2 \mathrm{~h}$ and $14 \mathrm{~h}$, respectively). Bedside echocardiography showed mild hypertrophy of the left ventricle with good overall systolic function (ejection fraction of $60 \%$ ) and mild pericardial effusion $(<5 \mathrm{~mm}$ ). At this point the initial ECG was reassessed. The ST-segment morphology in leads aVL, aVF, and V6 was considered highly unusual; however, the key observation was the lack of any ST segment changes only in lead II. This lead is recorded between electrodes attached to the right arm and left leg and it is the only lead that does not use the signal from the left arm electrode. Therefore, we hypothesised that the left arm must have been the source of artefacts. Because of chronic haemodialysis, the patient had a large arteriovenous fistula on the left forearm. On detailed examination, we noted prominent pulsation of the fistula, especially close to the patient's wrist (Suppl. Video 1 - see journal website). To confirm our hypothesis, we repeated ECG recordings. When the left arm electrode was placed on the pulsating fistula, we could replicate ischaemia-like artefacts in all limbs and precordial leads apart from lead II. The diagnostic workup was completed with a treadmill stress test, which was negative. Electrocardiographic motion artefacts are not uncommon in clinical practice. They may be caused by tremor (Parkinson's disease, thyrotoxicosis) or shivering (fever, hypothermia) [1]. Generally, these artefacts occur randomly throughout the ECG. The artefacts presented herein appeared in a constant relationship with the QRS complexes because they originated from arterial pulsation, which made the diagnosis challenging. We found one report of such a phenomenon in the literature, but it only included a recording of a single lead [2]. Recently, Aslanger [3] reported that radial artery pulsation could be a source of ST-segment elevation. Recognition of artefacts is an important part of ECG analysis in clinical practice. When ECG findings do not correlate with the clinical picture, follow-up ECGs and close attention to technical details of the ECG recording should be considered. In conclusion, we present the first case of arteriovenous fistula motion artefacts convincingly mimicking ACS.

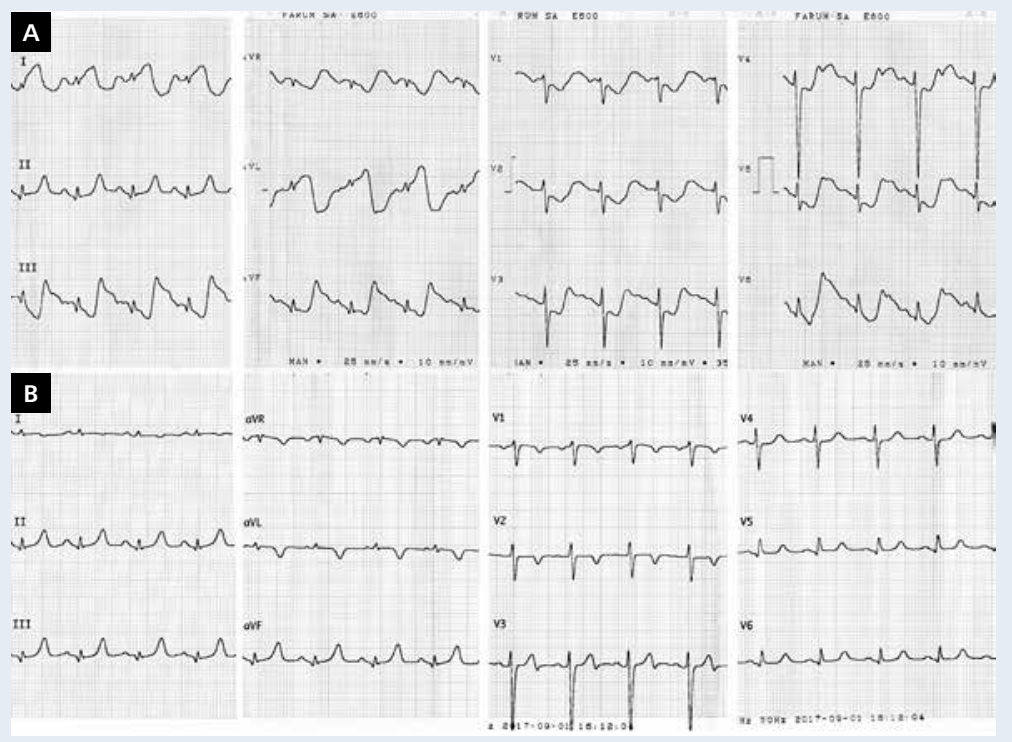

1. Pérez-Riera AR, Barbosa-Barros R, Daminello-Raimundo R, et al. Main artifacts in electrocardiography. Ann Noninvasive Electrocardiol. 2018; 23(2): e12494, doi: 10.1111/anec.12494, indexed in Pubmed: 28940924

2. Graziano VA, Leslie BR. Electrocardiographic artifact caused by an arteriovenous fistula. JAMA. 1979; 241(22): 2380, indexed in Pubmed: 439314.

3. Aslanger E. An unusual electrocardiogram artifact in a patient with near syncope. J Electrocardiol. 2010; 43(6): 686-688, doi: 10.1016/j.jelectrocard.2010.04.012, indexed in Pubmed: 20553822.

Figure 1. Initial electrocardiogram recorded in the neurology department (A). Follow-up electrocardiogram recorded in cardiac intensive care unit (B)

\section{Address for correspondence:}

Paweł Moskal, MD, $1^{\text {st }}$ Department of Cardiology, Interventional Electrocardiology, and Hypertension, Jagiellonian University Medical College, ul. Kopernika 17, 31-501 Kraków, Poland, tel: +48 1242473 00, fax: +48 1242473 20, e-mail: pawel@moskal.in

Conflict of interest: none declared

Kardiologia Polska Copyright (c) Polish Cardiac Society 2018 